\title{
Identification of multiple cyclin subunits of human P-TEFb
}

\author{
Junmin Peng Yuerong Zhu, Jeffrey T. Milton, and David H. Price ${ }^{1}$
}

Department of Biochemistry, University of Iowa, lowa City, Iowa 52242 USA

\begin{abstract}
The transition from abortive into productive elongation is proposed to be controlled by a positive transcription elongation factor $b$ (P-TEFb) through phosphorylation of the carboxy-terminal domain (CTD) of the largest subunit of RNA polymerase II. D rosophila P-TEFb was identified recently as a cyclin-dependent kinase (CDK9) paired with a cyclin subunit (cyclin T). We demonstrate here the cloning of multiple cyclin subunits of human P-TEFb (T1 and T2). Cyclin T2 has two forms (T 2a and T2b) because of alternative splicing. Both cyclin T1 and T2 are ubiquitously expressed. Immunoprecipitation and immunodepletion experiments carried out on Hela nuclear extract (HNE) indicated that cyclin T1 and T2 were associated with CDK9 in a mutually exclusive manner and that almost all CDK9 was associated with either cyclin T1 or T2. Recombinant CDK9/cyclin T1, CDK9/cyclin T2a, and CDK9/cyclin T2b produced in Sf9 cells possessed DRB-sensitive kinase activity and functioned in transcription elongation in vitro. Either cyclin T1 or T2 was required to activate CDK9, and the truncation of the carboxyl terminus of the cyclin reduced, but did not eliminate, P-TEFb activity. Cotransfection experiments indicated that all three CDK9/cyclin combinations dramatically activated the CMV promoter.
\end{abstract}

[Key Words: CTD; cyclin; CDK; RNA polymerase II; transcription; elongation; P-TEFb; DRB]

Received January 27, 1998; accepted February 3, 1998.

The postinitiation processivity of RNA polymerase II is believed to be controlled by both negative and positive (M arshall et al . 1996; Reines et al . 1996; Yamaguchi et al. 1998). N egative factors, such as factor 2 (Xie and Price 1996, 1997, 1998) and a 5,6-di chl oro-1- $\beta$-D-ribofuranosylbenzimidazole (DRB)-sensitivity inducing factor (DSIF) (Hartzog et al . 1998; Wada et al. 1998), cause premature stopping and termination of initiated polymerase resulting in the generation of only short abortive transcripts. Positive factors exemplified by positive transcription elongation factor $b$ (P-TEFb) promote the transition into productive elongation ( $M$ arshall and Price 1992, 1995; $M$ arshall et al. 1996). After entering productive elongation, the polymerase can be affected further by other elongation factors such as S-II, TFIIF, ELL, and el ongin to generate long transcripts (Reines et al. 1996).

$\mathrm{P}-\mathrm{TEFb}$ is proposed to facilitate the transition from abortive to productive elongation by phosphorylating the carboxy-terminal domain (CTD) of the largest subunit of RNA polymerase II (Marshall et al. 1996; J. Peng, N.F. Marshall, and D.H. Price, in prep). CTD phosphorylation is controlled by various kinases and phosphatases during different transcription stages (Dahmus 1994, 1995). RN A polymerase II has been found to be hypophosphorylated in preinitiation compl exes (Laybourn and Dahmus 1989) and in early elongation complexes in vitro (Marshall et

${ }^{1}$ Corresponding author.

E-MAIL david-price@uiowa.edu; FAX (319) 335-9570. al. 1996), but hyperphosphorylated during productive elongation (O'Brien et al. 1994; Laybourn and Dahmus 1989). Drosophila P-TEFb has been cloned and identified as a cyclin-dependent kinase pair (CDK9/cyclin T) that can phosphorylate the CTD in vitro (Peng et al ., in prep.). The kinase activity of P-TEFb is very sensitive to a purine analog, DRB (Marshall et al. 1996; Peng et al., in prep.). Consistently, the transition from abortive to productive el ongation can be inhibited by DRB in vitro and in vivo (Yamaguchi et al. 1998). Removal of the CTD in early elongation complexes abolished P-TEFb function, suggesting that the CTD is the target for P-TEFb function (Marshall et al. 1996).

The kinase activity of CDKs is regulated by phosphorylation and binding of cyclins and CDK kinase inhibitors (CKI) (M organ 1995). CDKs were first identified as regulators of the cell cycle but have now been implicated in other cellular events (M organ 1995). Association of a cyclin and phosphorylation of a conserved threonine residue in the T-Ioop are required to activate a CDK, whereas phosphorylation of specific threonine and tyrosine residues or association of CKIs can inactivate a CDK/cyclin complex (Morgan 1995). Although the se quences of CDKs are well conserved in the CDK family, the cyclins are not conserved except for a helix-rich cyclin box (Bazan 1996).

PITALRE (CDK9) was first cloned and identified as a CDK-like kinase with unknown function (Grana et al. 1994; Garriga et al. 1996). Recently, it was found to be a 
component of human P-TEFb that associated with HIV Tat and was required for HIV-1 Tat transactivation in vitro and in vivo (M ancebo et al. 1997; Yang et al. 1997; Zhu et al. 1997). Here we report the identification and cloning of multiple cyclin subunits of human P-TEFb. All three cyclins have been found associated with CDK9 in HeLa nuclear extract (HNE). Recombinant human PTEFb possesses strong CTD kinase activity and functions in transcription in vitro and in vivo.

\section{Results}

Cloning of three cyclin subunits of human P-TEFb

To further understand the function of P-TEFb in elongation control and in Tat transactivation, it was necessary to clone a human cyclin T subunit. After cloning Drosophila cyclin T (J. Peng, N.F. M arshall, and D.H. Price, in prep.), we searched the EST database for homologs. Three human sequences were found that led to the cloning of two related human genes: cyclins T1 and T2 (Fig. 1). Cyclin T2 had two forms termed T2a and T2b that likely arose because of al ternative splicing of the primary transcript. Cyclins T2a and T2b shared the first 642 amino acids but had different carboxyl termini. The cyclin box was highly conserved from Drosophila to human (Fig. 1B). In the cyclin box region, Drosophila cyclin $\mathrm{T}$ had $64 \%$ identity to either human cyclins T1 or T2, and human cyclins T1 and T2 shared $81 \%$ identity.

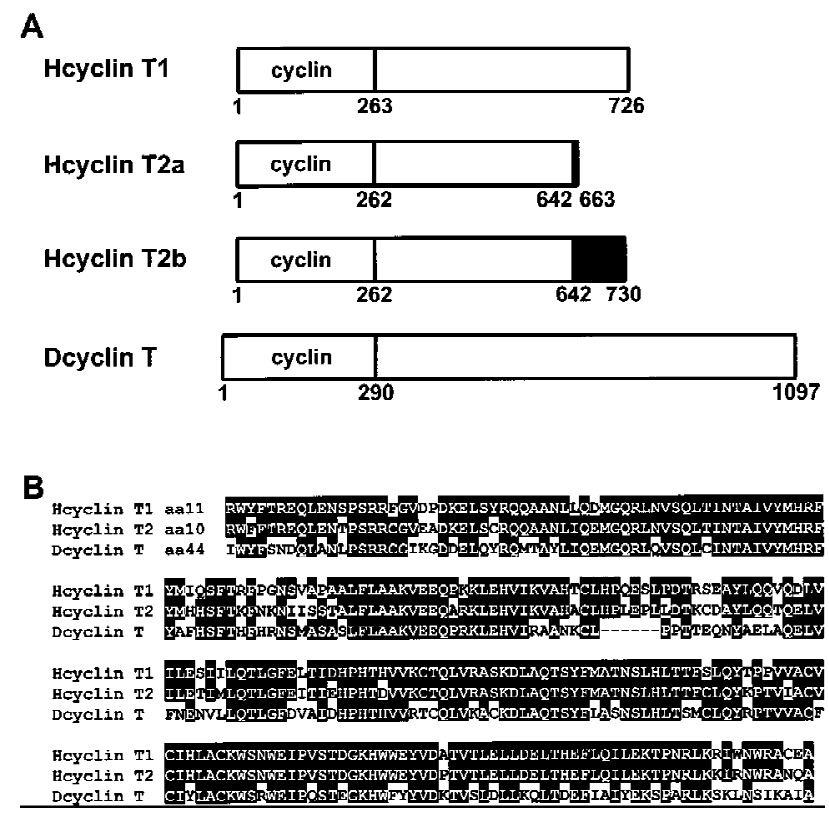

Figure 1. Sequence comparison of multiple human cyclin T's with Drosophila cyclin T. (A) Diagram of human cyclins T1, $\mathrm{T} 2 \mathrm{a}$, and $\mathrm{T} 2 \mathrm{~b}$ and Drosophila cyclin T. Amino acids are numbered on the bottom of each protein. The cyclin box is indicated. Human cyclins T 2a and T 2b have 642 amino acids in common but different carboxyl termini (black boxes). (B) Sequence alignment of the cyclin boxes. Identity is indicated by reverse shading.
However, the carboxyl terminus was much less conserved. In this region, Drosophila cyclin T showed $<25 \%$ identity to either human cyclin $\mathrm{T}$, whereas human cyclins T 1 and T 2 shared $\sim 46 \%$ identity.

Northern analysis indicated that human CDK9 and cyclins T1 and T 2 were expressed in all human tissues examined (Fig. 2). CDK9 had several mRNAs of 2, 2.7, and $3.4 \mathrm{~kb}$, similar to what was reported previously (Grana et al. 1994). Cyclin T1 had a major 8.2-kb mRN A in all tissues and a 9.3-kb mRN A in brain. Cyclin T 2 had a major $6-\mathrm{kb} \mathrm{mRNA}$ and a heterogeneous pattern of larger RN A species. The 6-kb band was not sharp suggesting the presence of more than one mRNA form. The ubiquitous expression of mRNAs encoding both subunits of P-TEFb was consistent with the general requirement of P-TEFb in transcription elongation.

\section{Multiple cyclin subunits exist and bind to CDK9}

cDNAs encoding CDK9 were coexpressed with either cyclin T1, T2a, or T2b in Sf9 cells (Fig. 3A), and recombinant proteins were purified using a nickel column followed by Mono S. The purified proteins were analyzed by SDS-PAGE (Fig. 3B). Only CDK9 was tagged, but the cyclin subunits were quantitatively retained on the nickel column indicating that the interaction of the cyclin and kinase subunit was strong. This was confirmed by the coelution of both subunits on the M ono S column. The interaction between the two subunits is, at least, partly due to the cyclin box region because a truncation mutant containing only the cyclin box of cyclin T2 [CDK9/T2 (1-286)]behaved similarly (Fig. 3).

To validate the cloning of appropriately sized cyclin subunits and to examine the natural abundance of the three potential forms of P-TEFb, antibodies were generated against the carboxyl terminus of either cyclin $\mathrm{T} 1$ or T2. Then, HNE was immunodepleted of either CDK9, cyclin T1, cyclin T2, or cyclin T1 and T 2 together. GST antibodies were used as a negative control. The immunoprecipitates (IPS) were analyzed by Western bl ot (Fig. 4A). As expected, CDK9 was present in the IP using antibodies against CDK9, cyclin T 1 and T2. Importantly, native cyclin $\mathrm{T} 1, \mathrm{~T} 2 \mathrm{a}$, or $\mathrm{T} 2 \mathrm{~b}$ proteins with identical mobilities to the recombinant proteins were detected. This indicates that all three forms are expressed in HeLa cells. Cyclin T1 was present in the IPS of CDK9 and cyclin T1 antibodies, whereas cyclin T2a and T2b were present in the IPs of CDK9 and cyclin T2 antibodies. These data strongly argued that CDK 9 can bind cyclin $\mathrm{T} 1$ or T2 but not both in one complex. Although the samples were loaded to reflect equivalent amounts of starting extract, the CDK9 detected in the IPs of cyclin T1 was about four times that in the IPs of cyclin T2, suggesting that the level of cyclin $\mathrm{T} 1$ was significantly higher than that of cyclin T2 in HNE.

To further demonstrate the association of CDK9 and the cyclins and quantitate the relative amount of cyclin T 1 and T2 in HNE, we performed a Western blot using various immunodepleted HNEs (Fig. 4B). Quantitation of the results was accomplished by loading different 

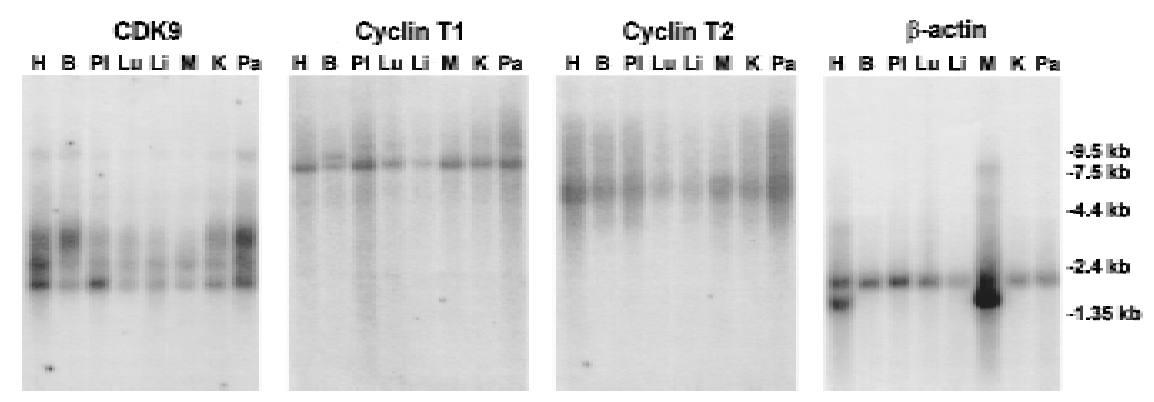

Figure 2. Expression pattern of human PTEFb subunits. A multiple tissue N orthern blot (Clontech) was probed according to the manufacturer. The sequences used for probes are as follows: (CDK9) Total encoding CDN A sequence; (cyclin T1) $0.4 \mathrm{~kb}$ encoding the amino terminus; (cyclin T2) $1.35 \mathrm{~kb}$ encoding the carboxyl terminus of cyclin T2b; $\beta$-actin: $2.0 \mathrm{~kb}$ of cDNA. The sizes of molecular markers are at right. $(\mathrm{H})$ Heart; (B) brain; (PI) placenta; (Lu) lung; (Li) liver; (M) skel etal muscle; (K) kidney; (Pa) pancreas.

amounts of the starting HNE from $1 \times$ to $10 \times$ Because THIIH was not found to be associated with P-TEFb, p62 (a subunit of TFIIH) was detected in all lanes and the signal correlated with the loading level of HNE (10× for all depleted HNEs). GST antibodies did not deplete any detected protein. In this experiment CDK9 antibodies depleted $85 \%$ of CDK 9 and $85 \%$ of cyclins T 1 and T 2 . T 1 antibodies depleted $65 \%$ of CDK $9,90 \%$ of cyclin T 1, but no cyclin T2. T2 antibodies depleted $20 \%$ of CDK9, no cyclin T 1 , and $90 \%$ of T2. T 1 , and T 2 anti bodies together depleted $75 \%$ of CDK9 and $85 \%$ of cyclins T1 and T2. These data strongly support that almost all CDK9 is associated with either cyclin T1 or T2. CDK9/cycl in T1 is the major form of the kinase, and no significant amount of free cyclin T or CDK9 exists in HNE. Furthermore, the similar signal detected for cyclins $\mathrm{T} 2 \mathrm{a}$ and $\mathrm{T} 2 \mathrm{~b}$ on the bl ot suggests that the 2 forms are present in equal amounts.

Recombinant P-TEFb proteins have kinase and transcription activities

Human P-TEFb possesses a CTD kinase activity that is sensitive to DRB (Zhu et al. 1997). To test the kinase activity of recombinant P-TEFb proteins, Drosophila RNA polymerase II was incubated with increasing amounts of recombinant proteins in the presence of 10 $\mu \mathrm{M}\left[\gamma^{-}{ }^{32} \mathrm{P}\right]$ ATP for $5 \mathrm{~min}$. The products were anal yzed by SDS-PAGE followed by autoradiography (Fig. 5A). CDK9/T 1 had slightly higher activity compared to both CDK9/T2a and CDK9/T2b, which had similar kinase activity to each other. All three proteins supported hyperphosphorylation of the large subunit of RNA polymerase II to the Ilo form. CDK9 al one had very little activity, and CDK9/T2 (1-286) had low activity and could not easily phosphorylate RNA polymerase II and cause a shift to the llo form. A wide-range titration experiment demonstrated that the kinase activity of CDK9/T2a was 10-20 times higher than CDK9/T2 (1286), at least 300 times higher than that of CDK9 alone (data not shown). The kinase activity of all three human P-TEFb proteins in the presence of increasing amounts of DRB was quantitated and plotted (Fig. 5B). The $50 \%$ inhibition point of CDK9/T1 $(0.9 \mu \mathrm{M})$ was similar to that of

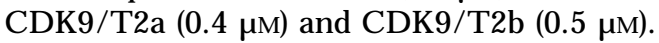

To analyze the function of recombinant P-TEFb proteins in transcription, they were added back to HNE depleted of CDK9. CDK9-depleted extracts have very little

A

a)

CDK9

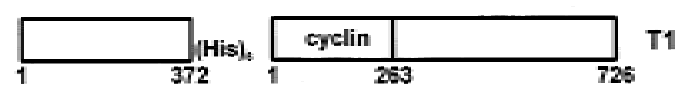

b)

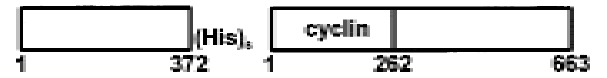

c)

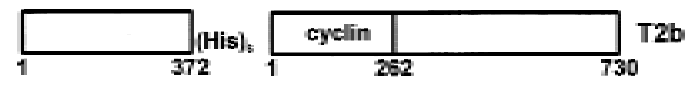

d)

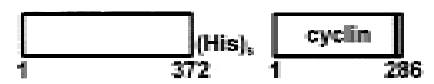

e)

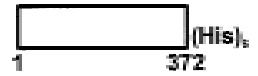

B

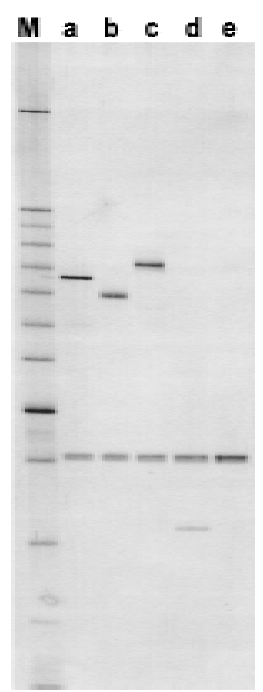

Figure 3. Purified recombinant human PTEFb proteins. (A) Diagram of P-TEFb protein constructs. (a) CDK9/T1; (b) CDK9/T2a; (c) CDK9/T2b; (d) CDK9/T2 (1-286); (e) CDK9. CDK9 is His-tagged. (B) SDS-PAGE (silver stained) of the purified recombinant proteins. (M) 10-kD ladder (10-120 plus 200 kD) with darker 50-kD band. 
Peng et al.

A

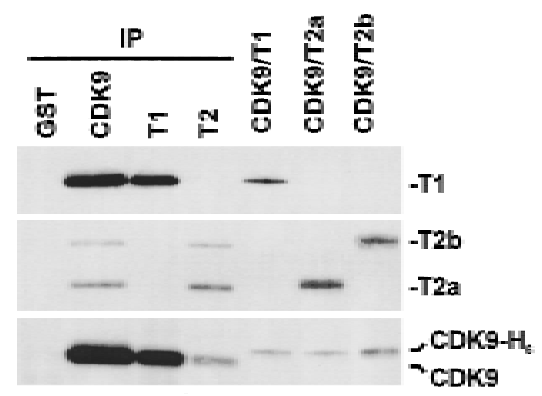

B

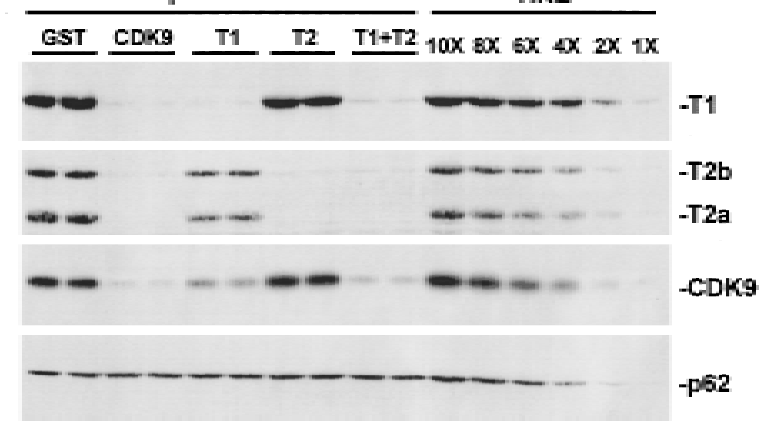

Figure 4. Multiple cyclin subunits are present and associated with CDK9 in HNE. (A) Western blot IPs. Human P-TEFb was immunoprecipitated using affinity-purified antibodies against GST, CDK9, cyclin T1, or cyclin T2. The IPs (equivalent to 10 $\mu \mathrm{HNE}$ ) were separated on an SDS gel and subjected to Western blotting using antibodies against cyclins T1, T2, or CDK9. The identity of the proteins is indicated at right. Recombinant proteins CDK9/T 1, CDK9/T2a, and CDK9/T2b were used as standards. (B) Western blot of immunodepleted HNE. HNE was depleted using antibodies against GST, CDK9, cyclin T1, cyclin T2, or cyclins T 1 and T2 together. Depleted HN Es equival ent to $2 \mu \mathrm{l}$ of HNE (10x) were loaded on an SDS gel followed by Western blotting using antibodies against cyclins T1, T2, CDK9, or p62 (a TFIIH subunit as a loading control). (Right) Original HNE was titrated as a quantitation standard.

DRB-sensitive transcription unless exogenous P-TEFb is added (Zhu et al. 1997). In this experiment a slight residual DRB sensitivity was detected in the depleted extract (Fig. 6A) that may be attributed to incomplete depletion. Addition of increasing amounts of recombinant P-TEFb proteins to the depleted extract restored DRB-sensitive transcripts to levels higher than that observed with the low level of endogenous P-TEFb in the original extract (Fig. 6). CDK9/T1 gave similar results to CDK9/T2a. Both of them had slightly higher activity than CDK9/T2b. CDK9/T2 (1-286) showed some activity, but CDK9 al one exhibited very little activity. The function of the recombinant proteins in transcription mirrored their ability to phosphorylate the CTD of RN A polymerase II, consistent with the idea that P-TEFb functions through the phosphorylation of CTD.

Overexpression of human P-TEFb activates the CMV promoter in vivo

To examine the effect of overexpression of P-TEFb in vivo HeLa cel Is were cotransfected with constructs containing the CMV promoter driving luciferase and constructs expressing CDK9 and the three cycl in T's (Fig. 7). Cotransfection with CDK9 increased the expression of CMV sixfold and cyclin T1 increased expression fourfold. These enhancements might be attributlable to the presence of uncomplexed CDK9 and T 1 in the cells being driven into complex by increasing the concentration of either partners. It is also possible that either subunit might titrate out an inhibitor of P-TEFb. The most dramatic stimulation of the CMV promoter occurred when both the kinase and a cyclin partner were added together. CDK9/T1 gave the greatest stimulation (38-fold) and CDK9/T $2 a$ and CDK9/T $2 b$ gave 26- and 16-fold stimulation, respectively. These results strongly suggest that even for one of the strongest promoters known, in vivo expression is limited by P-TEFb activity.

\section{Discussion}

We have identified three human cyclin CDN As encoding proteins $T 1, T 2 a$, and $T 2 b$, which activate CDK9. $T 1$ is the major form of the cyclin, and T2a and T2b are less abundant forms derived from a second gene. All three proteins are expressed in a wide variety of human tissues

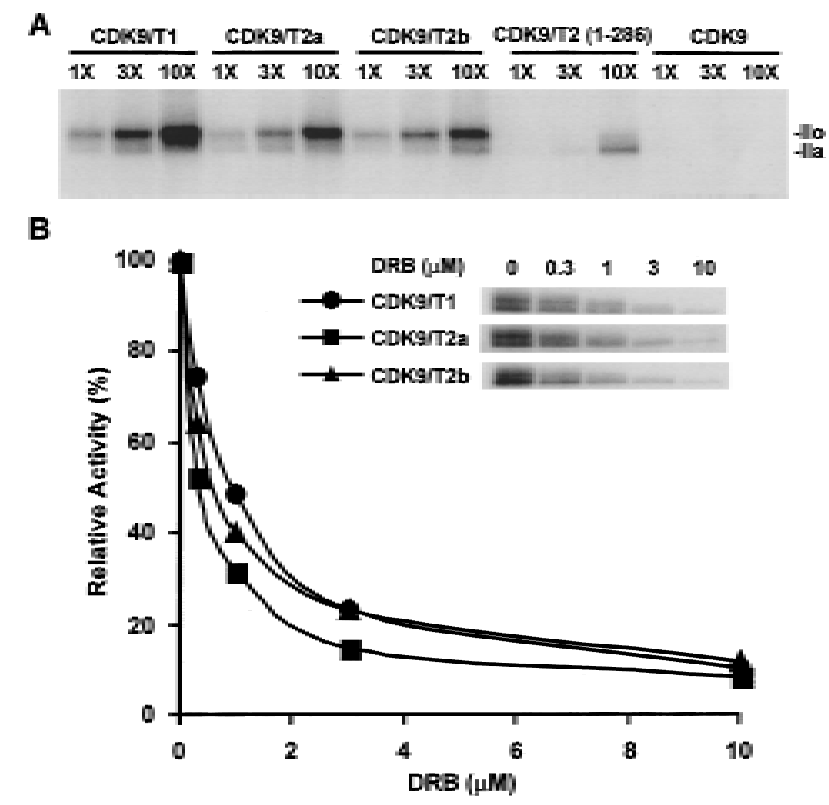

Figure 5. Recombinant human P-TEFb proteins have DRBsensitive CTD kinase activity. (A) CTD kinase assay using Drosophila RNA polymerase II and the indicated amount of recombinant proteins. Labeled RNA polymerase molecules were analyzed on a $6 \%-15 \%$ SDS-polyacrylamide gel by autoradiography. (1X) A pproximately $10 \mathrm{fmoles}$. (IIo and IIa) Shifted and unshifted largest subunit of RNA polymerase II, respectively. (B) DRB inhibition. P-TEFb proteins (5x) were used in the kinase assay with the addition of the indicated amount of DRB. Radiolabeled RNA polymerase II was quantified using a Packard Instantlmager and normalized to the starting level (100\%). 


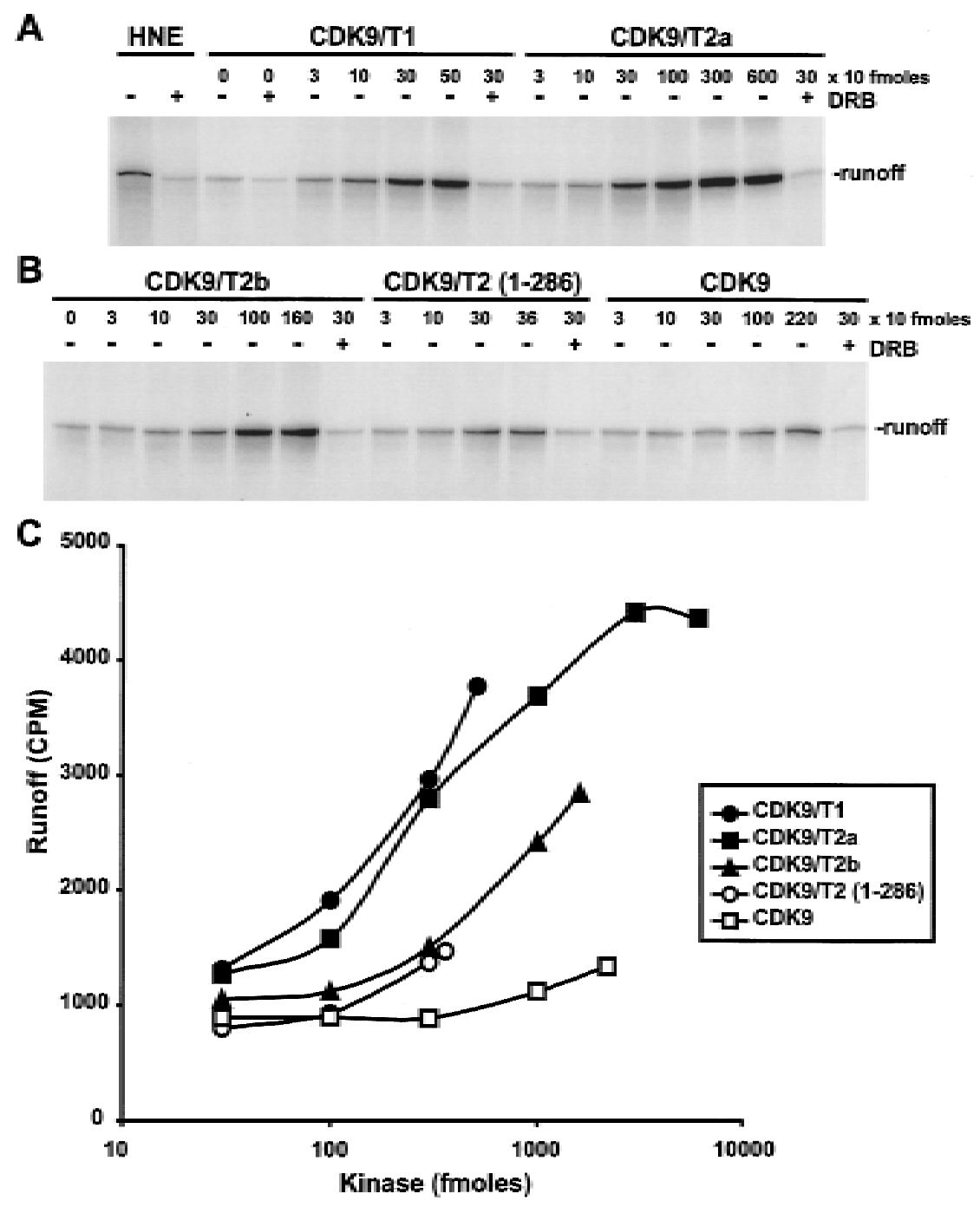

HNE 16030 $+$

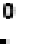
$100220 \quad 30 \times 10 f$
$-\quad+\quad+0 R B$ noff
Figure 6. Recombinant human P-TEFb proteins function in transcription. (A,B) Continuous labeling transcription using a CMV promoter was performed with HNE or CDK9-depleted HNE with addition of the indicated amount of recombinant proteins. DRB (50 $\mu \mathrm{m})$ was added as shown. (C) Plot of runoff. The radioactivity in the runoff transcripts was quantitated using a Packard InstantImager. and are found complexed with CDK9 in HeLa nuclear extract. Roughly $80 \%$ of the CDK9 is complexed with cyclin T1, $10 \%$ with T $2 \mathrm{a}$, and $10 \%$ with T $2 \mathrm{~b}$. Any of the three cyclin proteins complexed with CDK9 form an active P-TEFb moleculethat can phosphorylate the CTD of RNA polymerase II and cause the DRB-sensitive transition from abortive elongation into productive elongation.

Cyclins $T 2 \mathrm{a}$ and $\mathrm{T} 2 \mathrm{~b}$ are derived from the same gene but differ from each other after amino acid 642 . This difference is likely due to alternative splicing, a process known to produce variety in other cyclins. Two splice variants of cyclin E have been described. Cyclin Es lacks 49 amino acids within the cyclin box compared to cyclin E and cannot activate CDK2 (Sewing et al. 1994). Cyclin ET is missing 45 amino acids outside the cyclin box but, like Es, may not be functional (M umberg et al. 1997). A cyclin C splice variant has also been described that is truncated in the cyclin box ( $\mathrm{Li}$ et al. 1996). The two forms of cyclin T2 are both able to activate CDK9 and to function in transcription. Although it is possible that the

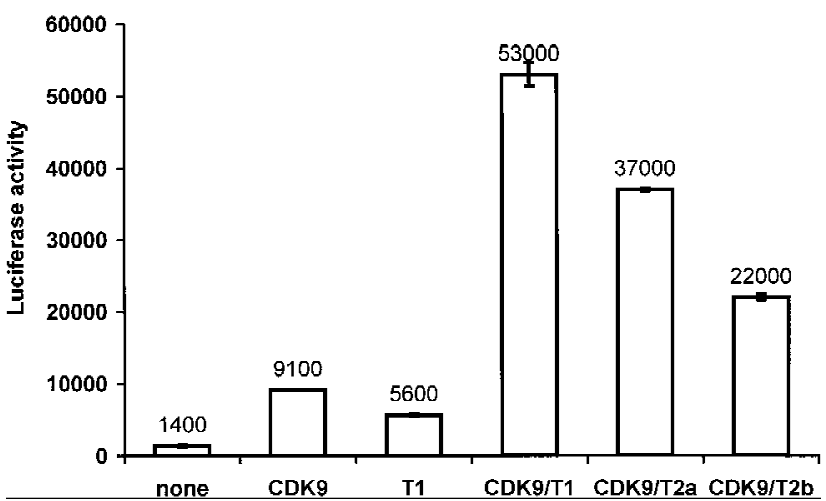

Figure 7. Overexpression of human P-TEFb in HeLa cells stimulates transcription from a CMV promoter. Transfections contained 1 million HeLa cells with $0.5 \mu$ g of a plasmid with the CMV promoter-driving luciferase as a reporter and $0.25 \mu \mathrm{g}$ of each plasmid with the CMV promoter-driving CDK9 or the indicated cyclin. Error bars are derived from comparing results from duplicate transfections. This experiment was performed a number of times and the results are typical. 
two forms play different roles in vivo, we have been unable to functional ly differentiate the two forms in vitro.

We showed here that $\mathrm{T} 1, \mathrm{~T} 2 \mathrm{a}$, and $\mathrm{T} 2 \mathrm{~b}$ individually form a complex with CDK9, but it is not clear whether other proteins are also functionally associated with CDK9 in vivo. Earlier results suggested the presence of a number of proteins associated with CDK9 (Grana et al. 1994; Zhu et al. 1997). Silver staining of CDK9 (PITALRE) immunoprecipitates showed proteins of 87 , 105, 133, 140, and 207 kD (Zhu et al. 1997). The 87-kD protein has now been identified as T 1 . T2a and T 2b are present in the immunoprecipitates (see Fig. 4) but are difficult to detect by silver staining the IPs because of their low abundance. The identity of the other proteins remains a mystery. Because the combination of CDK9 and either T1, T2a, or T2b forms an active P-TEFb protein, the potential role of the other proteins is not clear.

Our results extend the general finding that multiple cyclin subunits are found for a single CDK subunit (M organ 1997); however, different roles for the three cyclins have not been found. If the three cyclins do not have redundant functions it is possible that they have specific roles during the transcription of different genes. The CTD s of the T 1 and T 2 cyclins are significantly different from each other, and this domain in other cyclins has been found to interact with the proteins utilized as kinase substrates (M organ 1997). Supporting the rol e of the CTD in substrate binding we found that removal of this region of the $\mathrm{T} 2$ cyclin significantly reduced the ability of the CDK9/T 2 cyclin box protein to phosphorylate the CTD of RNA polymerase II and function in transcription. Besides the three cyclins, it is possible that there are other minor cyclin species in HeLa nuclear extract that can associate with CDK9.

The cloning and production of recombinant human P-TEFb should facilitate understanding the mechanism of DRB-sensitive transcription by contributing to the generation of a more defined system. It is becoming cl ear that controlling the el ongation phase of transcription involves the interaction of a number of positive and negative factors with the el ongation complex (Yamaguchi et al. 1998). P-TEFb is required for Tat transactivation of the HIV promoter both in vitro and in vivo (M ancebo et al. 1997; Zhu et al. 1997). Once a more defined system that exhibits elongation control has been developed it will be possible to examine the mechanism of T at transactivation in detail, and this in turn may lead to enlightened approaches to the blocking of Tat function.

It is possible that expression of human cyclin T1 (or T2) in rodent cells will allow more efficient Tat transactivation. $\mathrm{CHO}$ cells support only very low levels of Tat transactivation (Alonso et al. 1992, 1994). However, Tat transactivation is 10-fold higher in a $\mathrm{CHO}$ 12-cell line carrying human chromosome 12 (Alonso et al. 1992, 1994). It is very likely that cyclin T 1 is present in the CHO 12-cell because one of the EST s with high identity to T1 (yd48c03.s1) was mapped to $12 q 12$. As human PTEFb is required for Tat transactivation ( $M$ ancebo et al. 1997; Zhu et al. 1997), it is possible that expression of cyclin T1 in CHO cells will fulfill the chromosome 12 requirement and allow efficient Tat transactivation in the rodent cells. Furthermore, the expression of the appropriate cell surface receptors CD4 (Deng et al. 1997) and CCR5 (M CN icholl et al. 1997) in rodent cells, al ong with cyclin T1, may allow the cells to be infected by HIV. If this is the case, it opens the exciting possi bility of developing a mouse model for HIV infection using transgenic mice expressing human cyclin $\mathrm{T}$.

\section{Materials and methods}

Materials

$\left[\alpha^{-32}\right.$ P]CTP (3000 Ci/mmole) and [ $\gamma^{32}$ P]ATP $(6000 \mathrm{Ci} / \mathrm{mmole})$ were purchased from ICN. Ribonucleoside triphosphates were from Pharmacia-LKB Biotechnology. DRB (Sigma) was dissolved in ethanol to $10 \mathrm{~mm}$ and stored at $-80^{\circ} \mathrm{C}$. All other chemicals were reagent grade.

\section{Generation of an expression construct for human CDK9}

The coding region of the small subunit of human P-TEFb (HuCDK9, also called PITALRE) was amplified by Vent DNA polymerase using pET 21a-PITALRE (Zhu et al. 1997) as template, and two primers (5'-ACCCCTCCGGATAAATATGGCAAAGCAGTACG and 5'-AATCATGCTCGAGGAAGACGCGCTCAAACT). The amplified 1.2-kb fragment was digested with BspEl and Xhol and cloned into the pBAC4X-1 ( $N$ ovagen) to generate a plasmid (pBAC-HuCDK9) for expression of a 6-His-tagged human CDK9.

\section{Cloning of multiple cyclin subunits of human P-TEFb}

Using BLAST to search the EST database for homologs of Drosophila cyclin $\mathrm{T}$, we found three putative human cyclin T EST clones: nc70h05.r1, yd48c03.r1, and zr91f10.s1.

The nc70h05.rl and yd48c03.rl clones were found to be derived from the same gene, with the nc70h05.r1 sequence upstream of the yd48c03.rl sequence. The $5^{\prime}$ cDN A sequence was obtained using a M arathon cDN A Amplification Kit (Clontech), human bone marrow $M$ arathon-ready CDN A (Clontech) as template, and three primers based on nc70h05.r1 (5'-GGAGACAAGTATGTGCTACCTTGATGACA， 5'-GGAATTCGGGCTGCTCCTCCACTTTAG, 5'-GGAATTCGCTGCTGGAGCCACAGAA). Using the same kit and CDNA, the $3^{\prime}$ region was obtained using primers based on yd48c03.r1 (5'-GTGTCACTGAAAGAATACCG, and 5'-GGAATTCAGGTGGAGATAAAGCTGC). The total CDNA sequence was obtained by combining them. According to the length of the PCR products, the whole sequence was $2.8 \mathrm{~kb}$. We sequenced $2.4 \mathrm{~kb}$ and identified an intact open reading frame of $2.2 \mathrm{~kb}$ designed as cyclin T1. The coding sequence $(2.2 \mathrm{~kb})$ was cloned by RT-PCR. CDN A was synthesized using a primer (5'-GGAATTCTTACTTAGGAAGGGGTGGAAGTG) and HeLa total RNA. The coding sequence was amplified using eLON Gase (Life Technologies), the CDNA, and two primers (5'-GCTCTAGATAAATATGGAGGGAGAGAGGAA, and 5'-GGAATTCTTACTTAGGAAGGGGTGGAAGTGGTGGAGGAGGTT). Finally, the amplified sequence was cloned into the plasmid pBAC-HuCDK9 to produce a coexpression plasmid pBAC-HuCDK9-T1.

Based on the sequence from zr91f10.s1, the $5^{\prime}$ region of T2 CDNA was obtained using a 5' RACE kit (GIBCO) with three gene-specific primers (5'-TTCCCACCAATGCTTTCC, 5'-CCATCAGTTGATACAGGGATCT, and 5'-GGAATTCAGAAGGTTGTAAGATGC) and human brain poly(A)+ RNA as tem- 
plate. The 3' region of the total sequence was obtained by using a Marathon cDNA Amplification Kit (Clontech) with three gene-specific primers (5'-ACACACAGATGTGGTGAAATGTACCCA, 5'-GCATCTTACAACCTTCTG, and 5'-GGAATTCATGGAAAGCATTGGTGGGAAT) and a brain Marathonready $C D N A$. The total $C D N A$ sequence obtained was $4.5 \mathrm{~kb}$. The coding region of cyclin T2 was amplified by RT-PCR using Expand polymerase (Boehringer $M$ annheim) with two primers (5'-GGACTAGTATAAATATGGCGTCGGGCCGTG, and 5'GGAGATCTTACATGTTCATTCCTTGGG). The CDNA template used in the $P C R$ reaction was synthesized with a primer (5'-CCTCCACTACTGGTTTGCCTGG) from human brain poly $(A)^{+}$RN A. Interestingly, two related coding sequences were amplified: 2-kb (for cyclin T2a) and 2.2-kb (for cyclin T2b). These two sequences were al so ampl ified from HeLa total RN A. The 2.2-kb sequence contained a putative intron of $101 \mathrm{bp}$, which was not found in the 2-kb sequence. Both cyclin T2a and T 2 b were cloned in an expression plasmid, pBAC-HuCDK9, to generate plasmids pBAC-HuCDK9-T2a and pBAC-HuCDK9T2b.

To generate a carboxy-terminal truncation of cyclin T2, the coding sequence of cyclin T2a was digested with Xbal and EcoRI (an internal site in the coding sequence) and cloned into pBAC-HuCDK9 to generate pBAC-HuCDK9-T2 (1-286).

\section{Expression and purification of recombinant P-TEFb proteins}

All proteins were purified essentially as described (J. Peng, N.F. Marshall, and D.H. Price, in prep.), with the following modifications. Recombinant baculoviruses were generated using BaculoGold DNA (PharMingen) and five plasmids: pBACHuCDK9-T1, pBAC-HuCDK9-T2a, pBAC-HuCDK9-T2b, pBAC-HuCDK9-T2 (1-286), and pBAC-HuCDK9. Sf9 cells were infected with the virus, incubated for 2-3 days, and harvested by centrifugation. The cells were lysed for $1 \mathrm{hr}$ in a buffer (10 mM Tris- $\mathrm{HCl}$ at pH 7.6, $150 \mathrm{~mm} \mathrm{NaCl}, 2 \mathrm{~mm} \mathrm{M} \mathrm{MCl}, 1 \%$ Triton X-100, and $0.1 \%$ of a saturated solution of PMSF in isopropanol and $0.5 \mu \mathrm{g} / \mathrm{ml}$ of other protease inhibitors, aprotinin, leupeptin, pepstatin, chymostatin, and antipain. The cell lysate was centrifugated at $2000 \mathrm{~g}$ for $10 \mathrm{~min}$ and then added with $\mathrm{N} \mathrm{aCl}$ to $0.5 \mathrm{M}$ and imidazole to $5 \mathrm{~mm}$ followed by centrifugation at $250,000 \mathrm{~g}$ for $1 \mathrm{hr}$. A N i ${ }^{2+}$-NTA-agarose column (Qiagen) was loaded with the supernatant, washed with a wash buffer $(10 \mathrm{~mm}$ Tris- $\mathrm{HCl}$ at $\mathrm{pH} 7.6,0.5 \mathrm{M} \mathrm{N} \mathrm{aCl}, 5 \mathrm{mM}$ imidazole, PMSF), and eluted in several steps with elution buffers $(10 \mathrm{~mm}$ Tris- $\mathrm{HCl}$ at $\mathrm{pH} 7.6,0.5 \mathrm{M} \mathrm{N} \mathrm{aCl}, 20-200 \mathrm{~mm}$ imidazole, PMSF). The eluted fractions were analyzed by SDS-PAGE. The fractions containing the desired proteins were pooled, diluted, and loaded onto 1-ml $\mathrm{M}$ ono $\mathrm{S}$ columns. The columns were eluted with a linear gradient from 0.08-0.5 м HGKEDP (25 mM HEPES at pH 7.6, 15\% glycerol, 0.08-0.5 M KCl, $0.1 \mathrm{mM}$ EDTA, $1 \mathrm{~mm}$ DTT, PMSF). The recombinant proteins eluted at various salt concentrations: HuCDK9/T1, 180-230 mm; HuCDK9/T2a, 140-190 mm; HuCDK9/T2b, 190-240 mm; HuCDK9/T2, (1-286), 100-150 $\mathrm{mm}$; and HuCDK9, 100-150 mm. The HuCDK9/T2 (1-286) fraction was reconcentrated on a $\mathrm{M}$ ono $\mathrm{S}$ column using $350 \mathrm{~mm}$ step elution. All procedures of purification were carried out at $4^{\circ} \mathrm{C}$. The protein concentration of HuCDK9/T2a was determined by a Bio-Rad assay using BSA as a standard. Concentrations of other proteins were obtained by using a Bio-Rad GS 670 scanning densitometer to compare the HuCDK9 subunit in each preparation analyzed on a single silver-stained gel.

Production of cyclin T1 and T2 polyclonal antibodies

Recombinant GST-T1 (402-726) fusion protein was generated using a T 7 polymerase-dependent expression system essentially as described ( $M$ arshall et al. 1996). A sequence coding the carboxy-terminal portion (amino acids 402-726) of cyclin T1 was amplified and cloned into a GST expression plasmid (pET 21aGST) ( $M$ arshall et al. 1996). The fusion protein was expressed in DE3 cells, purified using a glutathione affinity column (Pharmacia), and used to immunize rabbits (Pocono Rabbit Farm). Antibodies against cyclin T1 were affinity-purified. Similarly, antibodies against cyclin T2 were produced, except that GST T 2a (440-663) fusion protein was used as an antigen. Both cyclins $\mathrm{T} 2 \mathrm{a}$ and $\mathrm{T} 2 \mathrm{~b}$ were recognized by these antibodies because they shared the sequence from amino acids 1 to 642 .

\section{CTD kinase assay}

The assay was performed as described (J. Peng, N.F. Marshall, and D.H. Price, in prep.) in a $20-\mu$ l reaction containing purified Drosophila RN A polymerase II ( $10 \mathrm{ng})$, various amount of kinases, $20 \mathrm{~mm}$ HEPES (pH 7.6), $5 \mathrm{~mm} \mathrm{M} \mathrm{gCl}, 55 \mathrm{~mm} \mathrm{KCl}, 10 \mu \mathrm{m}$ unlabeled ATP, and $2 \mu \mathrm{Ci}$ of $\left[\gamma^{-32} \mathrm{P}\right] \mathrm{ATP}(\mathrm{ICN})$ at $23^{\circ} \mathrm{C}$ for $5 \mathrm{~min}$. Reactions were terminated and analyzed by SDS-PAGE followed by autoradiography.

\section{Immunodepletion of HUP-TEFb}

Protein A-Sepharose beads (Sigma) were incubated with affinity-purified antibodies at $4^{\circ} \mathrm{C}$ for $30 \mathrm{~min}$, washed with a buffer containing $25 \mathrm{~mm}$ HEPES (pH 7.6), $0.6 \mathrm{M} \mathrm{KCl}, 0.5 \% \mathrm{NP}-40$, and $1 \%$ Triton X-100, followed by $180 \mathrm{~mm}$ HGKEDPB (HGKEDP buffer plus $0.1 \mathrm{mg} / \mathrm{ml}$ BSA), and packed into three columns. HNE (in 180 mm HGKEDP) was passed over the three columns successively. The flowthrough of the third column was used as depl eted HNE. The beads in these three columns were collected and washed with the wash buffer thoroughly. The associated proteins on the beads were eluted with SDS loading buffer and anal yzed by western blot using anti bodies against PITALRE carboxyl terminus (amino acids 352-372) and cyclins T1 and T2.

\section{In vitro transcription}

The transcription assay was performed in a $20-\mu l$ reaction with a continuous labeling procedure ( $M$ arshall and Price 1995). The CM V template $(20 \mu \mathrm{g} / \mathrm{ml}$, 631-nucl eotide runoff) was incubated with either HNE or depleted HNE in the presence of $20 \mathrm{~mm}$ HEPES (pH 7.6), $7 \mathrm{~mm} \mathrm{M} \mathrm{gCl} 2,55 \mathrm{~mm} \mathrm{KCl}, 200 \mu \mathrm{m}$ each of ATP, GTP, and UTP, $10 \mathrm{~mm} \mathrm{CTP}$, and $4 \mu \mathrm{Ci}$ of $\left[\alpha{ }^{32} \mathrm{P}\right] \mathrm{CTP}$ at $30^{\circ} \mathrm{C}$ for $20 \mathrm{~min}$. P-TEFb proteins and DRB (final contribution, $50 \mu \mathrm{m}$ ) were added as indicated. Label ed products were anal yzed on $6 \%$ denaturing gels followed by autoradiography.

Transient cotransfection assays

A reporter vector containing CM V-driven luciferase was generated by subcloning the CMV transcription templates into pGL2 basic (Promega) vector. All expression plasmids were made in pCDNA3 (Promega) and used to drive the expression of CDK9, cyclin T1, cyclin T2a, or cyclin T2b. Cotransfection assays were performed basically as described in the manual supplied with the lipofectamine reagent (GIBCO BRL). HeLa cells were aliquoted into 12 -well plates and incubated at $37^{\circ} \mathrm{C}$ with $5 \% \mathrm{CO}_{2}$. The dishes containing 1 million cells were $~ 80 \%$ confluent on the day of transfection. Cells were transfected with lipofectamine in serum-free DMEM. Complete DMEM was added to the cells $5 \mathrm{hr}$ after the transfection. Cells were harvested after $42 \mathrm{hr}$. After lysis the luciferase activity was determined using a Promega luciferase assay system. Transfections and luciferase determinations were both done in duplicate. 


\section{Acknowledgments}

This work was supported by the $\mathrm{N}$ ational Institutes of Health (grant GM 35500). The sequences of the cyclins have been deposited in GenBank under accession nos. AF048730-AF048732.

The publication costs of this article were defrayed in part by payment of page charges. This article must therefore be hereby marked "advertisement" in accordance with 18 USC section 1734 solely to indicate this fact.

\section{Note added in proof}

A nother group independently isolated a cDN A encoding cyclin $\mathrm{T} 1$ and showed that the cyclin interacted with HIV-1 Tat and increased the affinity of Tat for TAR RN A (Wei et al. 1998).

\section{References}

Alonso, A. D. Derse, and B.M. Peterlin. 1992. Human chromosome 12 is required for optimal interactions between $T$ at and TAR of human immunodeficiency virus type 1 in rodent cells. J. Virol. 66: 4617-4621.

Alonso, A., T.P. Cujec, and B.M. Peterlin. 1994. Effects of human chromosome 12 on interactions between Tat and TAR of human immunodeficiency virus type 1. J. Virol. 67: 65056513.

Bazan, J.F. 1996. Helical fold prediction for the cyclin box. Proteins 24: 1-17.

Dahmus, M.E. 1994. The role of multisite phosphorylation in the regulation of RNA polymerase II activity. Prog. Nucleic Acid Res. Mol. Biol. 48: 143-179.

Dahmus, M.E. 1995. Phosphorylation of the C-terminal domain of RN A polymerase II. Biochim. Biophys. Acta Gene Struct. Expression 1261: 171-182.

Deng, H.K., D. Unutmaz, V.N. KewalRamani, D.R. Littman. 1997. Expression cloning of new receptors used by simian and human immunodeficiency viruses. Nature 388: 296-300.

Garriga, J., X. Mayol, and X. Grana. 1996. The CDC2-related kinase PITALRE is the catalytic subunit of active multimeric protein complexes. Biochem. J. 319: 293-298.

Grana, X., A. De Luca, N. Sang, Y. Fu, P.P. Claudio, J. Rosenblatt, D.O. Morgan, and A. Giordano. 1994. PITALRE, a nuclear CDC2-related protein kinase that phosphorylates the retinoblastoma protein in vitro. Proc. Natl. Acad. Sci. 91: 3834-3838.

Hartzog, G.A., T. Wada, H. Handa, and F. Winston. 1998. Evidence that Spt4, Spt5, and Spt6 control transcription el ongation by RN A polymerase II in Saccharomyces cerevisiae. Genes \& Dev. 12: 357-369.

Laybourn, P.J. and M.E. Dahmus. 1989. Transcription-dependent structural changes in the $\mathrm{C}$-terminal domain of mammalian RNA polymerase subunit Ila/o. J. Biol. Chem. 264: 6693-6698.

Li, H., J.M. Lahti, and V.J. Kidd. 1996. Alternatively spliced cyclin C mRNA is widely expressed, cell cycle regulated, and encodes a truncated cyclin box. Oncogene 13: 705-712.

Mancebo, H.Y., G. Lee, J. Flygare, J. Tomassini, P. Luu, Y.R. Zhu, J.M. Peng, C. Blau, D. Hazuda, D. Price, and O. Flores. 1997. P-TEFb kinase is required for HIV Tat transcriptional activation in vivo and in vitro. Genes \& Dev. 11: 2633-2644.

Marshall, N.F. and D.H. Price. 1992. Control of formation of two distinct classes of RNA polymerase II elongation complexes. Mol. Cell. Biol. 12: 2078-2090.

- - 1995. Purification of P-TEFb, a transcription factor re- quired for the transition into productive elongation. J. Biol. Chem. 270: 12335-12338.

M arshall, N.F., J.M. Peng, Z. Xie, and D.H. Price. 1996. Control of RNA polymerase II elongation potential by a novel carboxyl terminal domain kinase. J. Biol. Chem. 271: 2717627183.

McNicholl, J.M., D.K. Smith, S.H. Qari, and T. Hodge. 1997. Host genes and HIV: The role of the chemokine receptor gene CCR5 and its allele. Emerging Infect. Dis. 3: 261-271.

Morgan, D.O. 1995. Principles of cdk regulation. Nature 374: 131-134.

_-_. 1997. Cyclin-dependent kinases: Engines, clocks, and microprocessors. Annu. Rev. Cell Dev. Biol. 13: 261-291.

Mumberg, D., M. Wick, C. Burger, K. Haas, M. Funk, and R. Muller. 1997. Cyclin ET, a new splice variant of human cyclin E with a unique expression pattern during cell cycle progression and differentiation. Nucleic Acids Res. 25: 2098-2105.

O'Brien, T., S. Hardin, A. Greenleaf, and J.T. Lis. 1994. Phosphorylation of RNA polymerase II C-terminal domain and transcriptional el ongation. Nature 370: 75-77.

Reines, D., J.W. Conaway, and R.C. Conaway. 1996. The RN A polymerase II general el ongation factors. Trends Biochem. Sci. 21: 351-355.

Sewing, A., V. Ronicke, C. Burger, M. Funk, and R. Muller. 1994. Alternative splicing of human cyclin E. J. Cell Sci. 107: 581-588.

Wada, T., T.T. Takagi, Y. Yamaguchi, A. Ferdous, T. Imai, S. Hirose, S. Sugimoto, K. Yano, G.A. Hartzog, F. Winston, S. Buratowski and H. Handa. 1997. DSIF, a novel transcription elongation factor that regulates RNA polymerase II processivity, is composed of human Spt4 and Spt5 homologs. Genes \& Dev. 12: 343-356.

Wei, P., M.E. Garber, S.M. Fang, W.H. Fischer, and K.A. Jones. 1998. A novel CDK9-associated C-type cyclin interacts directly with HIV-1 Tat and mediates its high affinity, loopspecific binding to TAR RNA. Cell (in press).

Xie, Z. and D.H. Price. 1996. Purification of an RNA polymerase II transcript release factor from Drosophila. J. Biol. Chem. 271: 11043-11046.

-_- 1997. Drosophila factor 2, an RN A polymerase II transcript rel ease factor, has DNA-dependent ATPase activity. J. Biol. Chem. 272: 31902-31907.

-_- 1998. Unusual nucleic acid binding properties of factor 2 an RNA polymerase II transcript release factor. J. Biol. Chem. 273: 3771-3777.

Yamaguchi, Y., T. Wada, and H. Handa. 1998. Interplay between positive and negative el ongation factors; drawing a new view of DRB. Genes Cells (in press).

Yang, X.Z., M.O. Gold, D.N. Tang, D.E. Lewis, E. Aguilarcordova, A.P. Rice, and C.H. Herrmann. 1997. TAK, an HIV TAT-associated kinase, is a member of the cyclin-dependent family of protein kinases and is induced by activation of perifheral blood lymphocytes and differentiation of promonocytic cell lines. Proc. Natl. Acad. Sci. 94: 12331-12336.

Zhu, Y.R., T. Peery, J.M. Peng, Y. Ramanathan, N. M arshall, T. Marshall, B. Amendt, M.B. Mathews, and D.H. Price. 1997. Transcription el ongation factor P-TEFb is required for HIV-1 Tat transactivation in vitro. Genes \& Dev. 11: 2622-2632. 


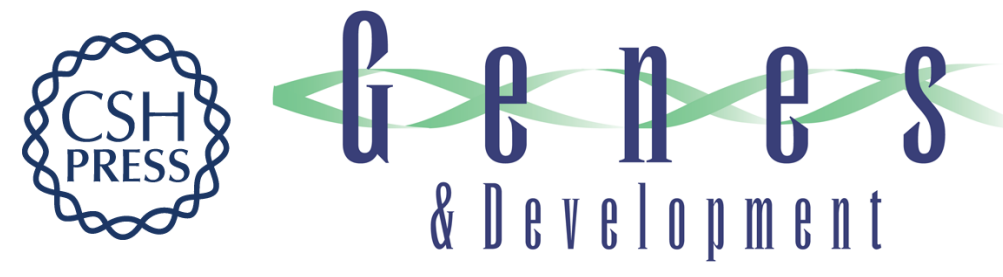

\section{Identification of multiple cyclin subunits of human P-TEFb}

Junmin Peng, Yuerong Zhu, Jeffrey T. Milton, et al.

Genes Dev. 1998, 12:

References This article cites 27 articles, 15 of which can be accessed free at: http://genesdev.cshlp.org/content/12/5/755.full.htmI\#ref-list-1

License

Email Alerting

Receive free email alerts when new articles cite this article - sign up in the box at the top Service right corner of the article or click here.

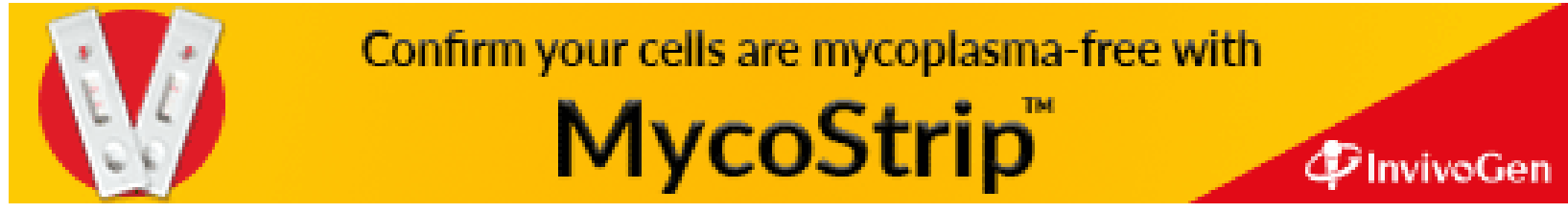

